\title{
Una aproximación a la situación de las mujeres en el Irán contemporáneo: perspectivas locales y globales
}

Alejandra Gutiérrez Luna*

Iraís Fuentes Arzate **

\section{Resumen}

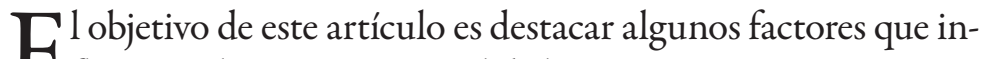
Cfluyen en la situación actual de las mujeres en Irán. Se sostiene que su posición en la sociedad puede ser entendida a partir de la influencia de factores locales y globales. Los locales son la ley de la familia, las características del sistema político iraní y las condiciones educativas y laborales. Respecto a los factores globales, se hace énfasis en las agendas de los organismos internacionales, las sanciones económicas impuestas por el gobierno estadounidense y los movimientos feministas trasnacionales. Dichos factores contribuyen a comprender bajo qué condiciones viven las mujeres en Irán y a ampliar la discusión que en algunos medios de comunicación se limita a cuestiones como la vestimenta.

\section{Palabras clave}

Género, Feminismo, Medio Oriente, Irán, Organismos Internacionales.

Fecha de recepción:

mayo de 2021
Fecha de aceptacion:

julio de 2021

Doctora en Ciencias Políticas y Sociales con orientación en Relaciones Internacionales. Profesora de la Facultad de Estudios Superiores Acatlán y Facultad de Ciencias Políticas y Sociales Universidad Nacional Autónoma de México. Contacto: alegutierrezluna@politicas.unam.mx ORCID: https:/orcid.org/0000-0002-9504-8837"

** Maestra en Relaciones Internacionales por la Universidad Autónoma Metropolitana Xochimilco (UAM-X). Contacto: blue.irais@gmail.com ORCID: https:/orcid. org/0000-0003-4822-8058 
160 UNA APROXIMACIÓN A LA SITUACIÓN DE LAS MUJERES EN EL IRÁN CONTEMPORÁNEO: PERSPECTIVAS LOCAlES y GlobAles / Alejandra Gutiérrez Luna, Iraís Fuentes Arzate

An Approach to the Situation of Women in Contemporary Iran: Local and Global Perspectives

Keywords

Gender, Feminism, Middle East, Iran, International Organizations.

\section{Abstract:}

This article aims to highlight some factors that influence the contemporary situation of women in Iran. It is argued that the position of women in Iran is understood by both local and global factors. Some local factors are family law, Iranian political system, and educational and working conditions. While global factors are the agendas of international organizations, economic sanctions imposed by the United States, and transnational feminist movements. These factors help to understand under which conditions women in Iran live and broaden the discussion that in some media is limited to questions of clothing.

\section{Introducción}

Con el título "la situación de las mujeres" no se busca generalizar o abstraer historias a partir de un discurso que pretende homogeneizar las diferencias de las mujeres en Irán; tampoco se trata de una aproximación orientalista que persigue culpar al islam de los problemas que atañen a las mujeres. Por el contrario, en este trabajo "la situación de las mujeres" refiere a una realidad material que depende de factores económico-políticos tanto internos como externos, los cuales conforman una estructura que asegura y perpetúa la subordinación y que, de ninguna forma, es exclusiva del régimen iraní, sino que forma parte de una estructura patriarcal global.

Con frecuencia, fuera de Irán las investigaciones sobre mujeres iraníes suelen tomar como punto de partida la Revolución Islámica de 1979, ya que se considera que a partir de dicho proceso muchas de las leyes y políticas, tanto en el ámbito público como en el doméstico, quedaron bajo el control directo de un sistema político islámico que ha promovido la discriminación de género 
a favor de los hombres (Tohidi, 2016, p. 78). Sin embargo, este es un argumento sesgado, ya que el régimen anterior, la monarquía Pahlaví (1925-1979), no consiguió alterar la estructura patriarcal de dominación, por lo que las mujeres no experimentaron mejoras en sus libertades y derechos civiles, sino que se utilizó a las mujeres para promover la imagen de una nación Occidental.

En este escrito se argumenta que la situación de las mujeres en Irán puede ser entendida a partir de la influencia de factores locales y globales. Los locales hacen referencia a la ley de la familia, las características del sistema político iraní y las condiciones educativas y laborales. Respecto a los factores globales, se hace énfasis en las agendas de los organismos internacionales, las sanciones económicas impuestas por el gobierno estadounidense y los movimientos feministas trasnacionales.

El objetivo del artículo es destacar algunos factores que influyen en la situación actual de las mujeres en Irán, y analizar de qué manera la relación entre elementos internos y externos ha impactado en la condición de las mujeres iraníes en la última década, para lo cual el escrito se divide en dos partes: la primera se enfoca en el estudio de los factores locales y la segunda en los elementos globales.

\section{La influencia de los factores locales en la situación de las mujeres en Irán: el caso del sistema político iraní}

La República Islámica de Irán, fundada tras la revolución iraní de 1979, es un sistema político dividido en órganos electivos y no electivos subordinados al Rabbar o Líder, siendo los no electivos los de mayor autoridad y en los que se han posicionado las facciones más conservadoras de la clase política iraní. "La combinación entre la estructura clerical chií-iraní y el republicanismo ha convertido a cierto sector de los Roubaniyat (clérigos) iraníes en la élite política por excelencia de todo el sistema político" (Zaccara, 2006, p. 27).

El Estado iraní, al igual que el resto de los Estado-nación engranados en la economía-mundo capitalista, es una estructura 
patriarcal que perpetúa la subordinación de las mujeres a través de instituciones y mecanismos como la Constitución de 1979 (reformada en 1989), el Código Civil y la Ley de la Familia, así como también el órgano no electivo del Consejo de Guardianes. En consecuencia, las mujeres enfrentan diversas formas de discriminación en su vida cotidiana: de por vida están bajo la tutela de los hombres de su familia, pues legalmente requieren el permiso de sus esposos o padres para trabajar y viajar al extranjero; las mujeres no pueden ejercer la profesión de juezas —únicamente se desempeñan como asesoras de los jueces especialmente en la corte de lo familiar, aunado a que el testimonio de las iraníes en una corte equivale a la mitad que el de un hombre-; los iraníes tienen derecho a divorciarse unilateralmente de su esposa y, tras el proceso de divorcio, las mujeres pierden la custodia de sus hijas a los siete años y la de sus hijos a los dos años (Osanloo, 2009).

El Estado patriarcal implica que la desigualdad, la violencia y la segregación por género atraviese todas las instituciones, discursos, leyes, prácticas, medidas administrativas; en suma, la totalidad de las relaciones sociales. Históricamente, las mujeres iraníes organizadas han luchado por sus libertades y derechos civiles; no obstante, el Consejo de Guardianes se ha encargado de frenar los cambios en materia de género. Se trata de la segunda institución más poderosa del sistema político iraní (tan solo después de la oficina del Líder Supremo), cuyas facultades establecidas en la constitución son: 1) la función legislativa de cámara alta que ratifica las leyes emanadas del Maylis o Parlamento, 2) la interpretación de la Constitución, y 3) la supervisión de las elecciones presidenciales, parlamentarias y referendos (Zaccara, 2006).

Desde su instauración en 1979, este órgano está controlado por fuerzas conservadoras que a menudo actúan para evitar un cambio legislativo que favorezca la equidad de género (Sephri, 2017). ${ }^{1}$ El Consejo de Guardianes se ha constituido en la prin-

1 La categoría de "equidad de género" se refiere a la búsqueda la igualdad de oportunidades para las personas y al desarrollo de las capacidades básicas, lo cual implica la eliminación de las barreras que obstaculizan las oportunidades económicas, políticas y sociales para las mujeres (Navas, 2018). 
cipal arma de las facciones más conservadoras de la República para bloquear las reformas en materia de género propuestas por el Maylis, como la Ley de la Familia, reformas al código civil, la participación de más mujeres en las instituciones estatales, la reforma al código penal, entre otras. De esta suerte, entre 2000 y 2004 las parlamentarias reformistas presionaron audazmente a funcionarios y clérigos de alto rango para cambiar las leyes a favor de las mujeres, incluida la ratificación de la Convención sobre la Eliminación de todas las formas de Discriminación contra la Mujer (CEDAW, por sus siglas en inglés), iniciativa que fue retomada en el marco de las elecciones presidenciales de 2009; no obstante, este y otros proyectos orientados a promover las causas de las mujeres fueron bloqueadas por el Consejo de Guardianes en ambos períodos (Bahramitash, 2007).

Durante la administración presidencial del principialista Mahmud Ahmadineyad (2005-2013), los conservadores impulsaron la "cultura de la modestia", una campaña encabezada por mujeres Basiy ${ }^{2}$ destinada a restaurar el uso estricto del hiyab y la vestimenta islámica que, desde la interpretación de hombres conservadores, se había salido de control durante el periodo de reforma (1997-2005) (Sadeghi, 2009). Además, los conservadores hicieron cumplir la prohibición de la grabación de voces femeninas; las mujeres fueron excluidas de más de 70 asignaturas de grado en el año académico de 2012, y se impuso el control sobre los viajes de mujeres solteras hasta los 40 años (Sreberny, 2013). No obstante los esfuerzos del gobierno conservador, el uso del hiyab es una práctica que se mantiene viva, varía del uso tradicional a colores y estilos llamativos que difícilmente tendrán retrocesos en función de los deseos de los hombres conservadores del régimen.

\footnotetext{
2 Los Basiy son un ejército de voluntarios conformado el 1980 por el Ayatolá Ruhollah Jomeini para sostener la guerra Irán-Iraq (1980-1988). Los Basiy consiguieron subsistir a pesar del término de la guerra en 1988 y se institucionalizaron como fuerzas de orden. En la actualidad, son el aparato responsable del control de disturbios y de la seguridad interna. Sus miembros generalmente actúan como "defensores locales" en pueblos y ciudades, monitorean la actividad de los ciudadanos, recaban información y, algunos batallones, se encargan de reprimir manifestaciones populares (Golkar, 2015).
} 
A pesar de que los reformistas han sido los más interesados en impulsar cambios legales para mitigar las brechas de género, existe un consenso entre los políticos iraníes para perpetuar la dominación masculina y el sistema patriarcal. "Los políticos iraníes han considerado que la cuestión de las mujeres y sus derechos legales y de ciudadanía no están entrelazados con la construcción de la democracia, y por lo tanto no constituyen un tema urgente para las ideologías democráticas" (Kian, 2005, pp. 57-58).

Muchos partidos, facciones y organizaciones políticas han utilizado las causas de las mujeres para la toma del poder, o han utilizado el cuerpo y la vestimenta de las mujeres como marcadores públicos de sus reclamos de identidad; pero una vez en el poder, las élites patriarcales han negado a las mujeres cualquier influencia, reforma o participación real que favorezca a sus intereses (Tajali, 2015). Los ejemplos van desde Reza Shah, Mohammad Reza Pahleví, el Ayatolá Ruhollah Jomeini, los expresidentes Mohammad Jatamí, Mahmud Ahmadineyad y Hasán Ruhaní, por citar algunos. Históricamente, los cambios tangibles en materia de género los han conseguido las mujeres organizadas como la reforma a la ley de la familia en 2007, la ley de sucesiones, el derecho penal, reformas al contrato matrimonial y el divorcio, entre otros.

\section{El Código Civil y la Ley de la Familia}

Uno de los discursos más recurrentes sobre el Irán posrevolucionario es que las mujeres perdieron gran cantidad de derechos y libertades civiles con la revolución de 1979. Si bien durante la era Pahlaví (1925-1979), el gobierno monárquico cedió a las presiones del movimiento feminista liberal, lo cierto es que utilizó la imagen de las mujeres como instrumento de representación de una nación secular-progresista. Por ejemplo, en 1936 el Shah ordenó a las mujeres que se quitaran el velo para promover una imagen de una "nación desvelada"; impulsó una campaña de planificación familiar desde arriba comprendida en la Ley de Protección de la Familia $^{3}$ (1967 y 1975) y permitió el acceso al aborto. En 1963

3 La Ley de Protección de la Familia, puesta en vigor en 1967 bajo el gobierno de 
concedió el derecho al voto femenino. Sin embargo, desde 1953 el Shah consolidó una dictadura real y los cargos gubernamentales de alto rango no estaban sujetos a votación, por lo que no representó un cambio importante (Kian, 2013). A pesar de que la dinastía Pahlaví se esforzó en atribuirse las libertades y derechos conseguidos por las mujeres organizadas en el período prerrevolucionario, lo cierto es que estuvo lejos de alterar el orden y la cultura patriarcal en Irán (Vakil, 2011), ya que las mujeres continuaron siendo consideradas ciudadanas de segundo orden.

Después de la revolución y la consolidación del poder republicano, los clérigos conservadores movilizaron la imagen de las mujeres como portadoras simbólicas de la virtud y el honor de ser esposas, madres y representantes de la castidad nacional, por lo tanto, "necesitaban ser salvadas" de la corrupción occidental (Osanloo, 2009). A partir de entonces, las leyes y políticas públicas en materia de género asumen a las mujeres como sinónimo de la "familia tradicional", por lo que constantemente agravian sus derechos y libertades civiles. En cambio, la Ley de la Familia se ha constituido en un mecanismo legal-institucional de subordinación.

Tan pronto fue derrocada la monarquía Pahlaví en 1979, el ayatolá Ruhollah Jomeini y sus seguidores derogaron la Ley de Protección de la Familia y, en su lugar, el Código Civil fue reformado en aras de integrar una serie de normas de conducta relacionadas con la definición de "familia islámica". Además, durante la década de 1980 impuso el uso obligatorio del hiyab y restringió los empleos formales remunerados para las mujeres: las juezas fueron destituidas de sus cargos, muchas trabajadoras del sector público fueron jubiladas prematuramente y diversas mujeres fueron excluidas de carreras universitarias como derecho, administración, ingeniería, jurisprudencia islámica, por mencionar algunas

Mohammad Reza Pahlaví y reformada en 1975, modificó sustancialmente algunas de las desigualdades inherentes al derecho de familia islámico. Abolió el derecho unilateral de los hombres a divorciarse e impulsó medidas más equitativas entre hombres y mujeres en materia de divorcio y custodia de los hijos. La ley de 1975 abolió el privilegio de divorcio para el marido e introdujo un reclamo de manutención postmatrimonial, entre otras cuestiones. 
166 UNA APROXiMACión A LA SITUACIÓN DE LAS MUJERES EN EL IRÁN CONTEMPORÁNEO: PERSPECTIVAS locales y Globales / Alejandra Gutiérrez Luna, Iraís Fuentes Arzate

(Kian, 2013). Aun cuando el régimen se esforzó por mantener a las mujeres fuera de la mirada pública, la guerra Irán-Iraq (19801988) y la posterior era de reconstrucción del país (1989-1997) requirió de la presencia y el trabajo remunerado y no remunerado de las mujeres iraníes.

A pesar de su integración en el ámbito económico, la desigualdad socioeconómica contra las mujeres ha persistido, debido al desinterés de los gobernantes por adoptar una agenda que mitigue la violencia estructural contra las mujeres; en cambio, las brechas de género están institucionalizadas en las leyes iraníes, por ejemplo, en materia de lo familiar. Mehrangiz Kar y Golriz Farshi (2008) resumen la ley de la familia en los siguientes puntos:

- La edad mínima para contraer matrimonio es de 15 años para las mujeres, mientras que para los hombres aumenta a los 18 años.

- En el caso de un asesinato, el dinero ensangrentado para una mujer es la mitad que el dinero correspondiente a un hombre.

- En la mayoría de los casos, el testimonio de una mujer no es válido en un tribunal de justicia.

- Una mujer no puede salir del país sin la aprobación de su esposo.

- Una mujer está legalmente obligada a ser obediente a su esposo.

- Un hombre puede tomar más de una esposa, incluso sin el consentimiento de la primera cónyuge.

- Un hombre puede prohibir el empleo de su esposa.

- Un hombre tiene el derecho inequívoco al divorcio (p. 84).

En lo que atañe al divorcio iniciado por una mujer, la Ley de la Familia estipula como indispensable demostrar que las penurias son insoportables para la esposa y perturban la vida matrimonial en un verdadero sentido conyugal, de modo que ella no pueda cumplir con sus propios deberes matrimoniales (Yassari, 2002). Solo en caso de que la esposa presente pruebas ante la corte de 
lo familiar se le concederá el divorcio. La mayoría de las veces la aprobación de la solicitud de divorcio iniciado por las mujeres depende de los jueces y su interpretación acerca de los motivos para solicitar la disolución del matrimonio. Debido a las dificultades a las que se enfrentan para separarse de sus esposos, muchas mujeres terminan renunciando a su $\mathrm{mahr}^{4}$ o dote, con tal de que su esposo firme a favor de la desintegración del vínculo marital (Mir-Hosseini, 1993).

En lo sucesivo, las mujeres divorciadas se enfrentan a una serie de problemas, empezando por la custodia de los hijos —una mujer tiene derecho a la custodia de sus hijos hasta los dos años y de sus hijas hasta los siete años-, el empleo, la remuneración —en Irán los maridos no están obligados a pagar una pensión alimenticia ni a su exesposa ni a sus hijos-, aunado a los prejuicios de una sociedad patriarcal que discrimina a una mujer divorciada.

En 2007, bajo la administración de Mahmud Ahmadineyad, el gobierno preparó un nuevo proyecto de Ley de la Familia que introdujo más restricciones a los derechos de las mujeres, como la eliminación de cualquier requisito de matrimonios temporales (sigheh) que elimina toda protección financiera o legal para las mujeres en estas uniones, el fomento de la poligamia, el impuesto sobre el mahr o dote de la novia que refuerza el poder financiero del marido durante el matrimonio, además de una serie de impedimentos procesales adicionales al divorcio cuando es solicitado por las mujeres (Kian, 2012).

Frente a este nuevo conjunto de políticas y tendencias regresivas, algunas defensoras de los derechos de la mujer optaron por la solidaridad de género y, más allá de sus divisiones políticas, se unieron y trabajaron juntas para oponerse al Proyecto de Ley de Familia. Argumentaron que, en su forma actual, el proyec-

4 Mahr consiste en una suma de dinero o algo de valor que el novio debe entregar a la novia al contraer matrimonio. La centralidad del mahr en el contrato matrimonial es tal que no puede haber matrimonio sin él. En algunos países, como Irán, no se produce ninguna transferencia de riqueza en el momento del matrimonio y, en la práctica, el mahr solo se paga si se produce el divorcio (Mir-Hosseini, 1993). 
to de ley sería inevitablemente perjudicial para los intereses y derechos de las mujeres y presentaron propuestas para cambiar las controvertidas disposiciones del proyecto de ley. Tras discusiones en profundidad con los miembros del comité judicial y otros parlamentarios, y aprovechando las rivalidades entre el presidente Ahmadineyad y el presidente del Maylis, Alí Larijani, lograron convencer a la mayoría de los parlamentarios para que retiraran los dos controvertidos artículos (artículos 22 y 25) que atentaban contra la libertad de las mujeres (Kian, 2012, pp. 67-68).

Este no es un caso excepcional en el que las mujeres consiguen avances en materia de género, pues tradicionalmente las iraníes han cuestionado las leyes islámicas que otorgan a los hombres de su familia el derecho de administrar sus cuerpos y sus vidas, han objetado la tradición de los jurisconsultos de decidir arbitrariamente el lugar y la posición que ocupan en la sociedad, y han demandado sus derechos a ocupar los mismos cargos públicos que los hombres.

\section{La Situación de la Educación y Empleo para las Mujeres en Irán}

Desde la década de 1980 las mujeres iraníes han logrado conquistar cada vez más espacios en las escuelas y universidades. Durante la misma década el analfabetismo femenino disminuyó progresivamente derivado del programa de alfabetización para mujeres impulsado por Ruhollah Jomeini, lo cual brindó a las mujeres feministas y de izquierda la oportunidad de participar en programas para mitigar la desigualdad de género (Bahramitash y Esfahani, 2011). De acuerdo con el Índice Global de Brecha de Género 2020, Irán ocupa el lugar número 118 de 153 países con respecto a los logros en educación para las mujeres; un lugar admisible y en marcada diferencia con otros indicadores que evalúan la desigualdad de género como el empleo (Irán ocupa el lugar 147 de 153 países), la salud (puesto 130 de 153) y el empoderamiento político (puesto 145), en los cuales Irán se encuentra entre los últimos seis lugares de la lista a nivel mundial (WEF, 2020). 
En materia educativa, las iraníes se han posicionado como una de las sociedades con la mayor tasa de alfabetización y escolaridad a nivel regional. Tal como lo representa la figura 1, para 2016 la tasa bruta de matriculación de mujeres en universidades alcanzó el 64.3\%. Esta tendencia está modificando la estructura de la familia, al incrementar la edad en que las iraníes suelen contraer nupcias y al permitirles alejarse de las funciones reproductivas obligatorias.

Figura 1. Índice de participación universitaria en Irán

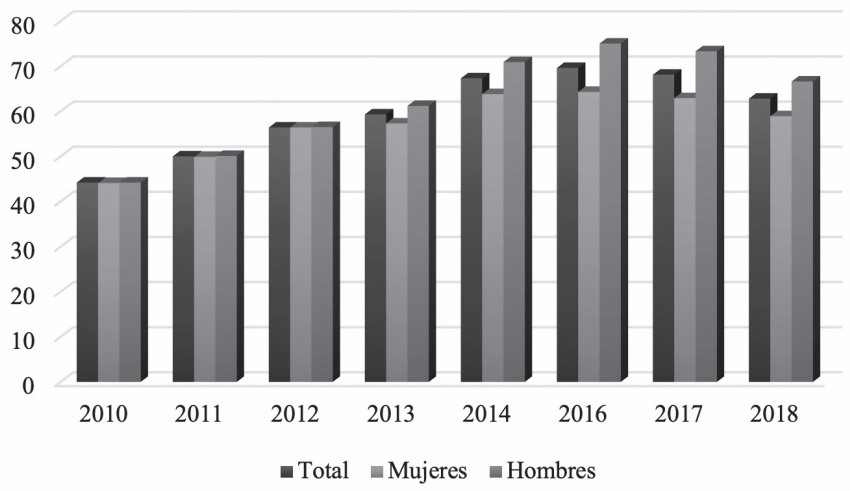

Elaboración propia con información de Banco Mundial, 2021.

No obstante, en Irán el sistema educativo se encuentra altamente centralizado, por lo que la planificación estratégica y la regulación educativa dependen de las políticas gubernamentales y son los hombres conservadores que toman las decisiones en este ámbito. Su enfoque patriarcal se ve agravado por la existencia del Consejo Superior de la Revolución Cultural, órgano encargado de presidir la islamización de las universidades y el sistema educativo en general, creado tras la revolución de 1979 (Aryan, 2016). En la actualidad, las mujeres universitarias representan más del $50 \%$ de la matrícula escolar. Sin embargo, los espacios educativos están bastante lejos de ser equitativos en términos de género, ya que las estudiantes se enfrentan a una serie de violencias en los campus universitarios, como la segregación por género, la exclu- 
170 UNA APROXIMACIÓN A LA SITUACIÓN DE LAS MUJERES EN EL IRÁN CONTEMPORÁNEO: PERSPECTIVAS locales y Globales / Alejandra Gutiérrez Luna, Iraís Fuentes Arzate

sión de muchos deportes y actividades recreativas; la restricción de las plazas para mujeres en ciertas carreras, y el acoso de los cuerpos de seguridad que se aseguran de que las mujeres cumplan con códigos de vestimenta estrictos.

Debido a que el aumento significativo de mujeres universitarias perfila un cambio en el mercado laboral, el Estado ha impuesto un sistema de cuotas que ofrece el 60\% de las plazas universitarias a los estudiantes y el $40 \%$ a las estudiantes (Nejadbahram, 2016), una política patriarcal aplicada durante 2005, 2007 y 2008 bajo la administración de Mahmud Ahmadineyad.

Aunado a lo anterior, al egresar de escuelas y universidades, las iraníes se encuentran con un panorama desalentador y sumamente desfavorable para su desarrollo personal y profesional, pues el mercado laboral se encuentra perfilado a partir de características masculinas e instituciones patriarcales. Como lo expresa la figura 2, en 2020 el desempleo masculino representó el 9.2\%, mientras que el femenino duplicó la cifra con $18.9 \%$, una tendencia que sigue empeorando por la crisis económica.

Figura 2. Indice de desempleo en Irán

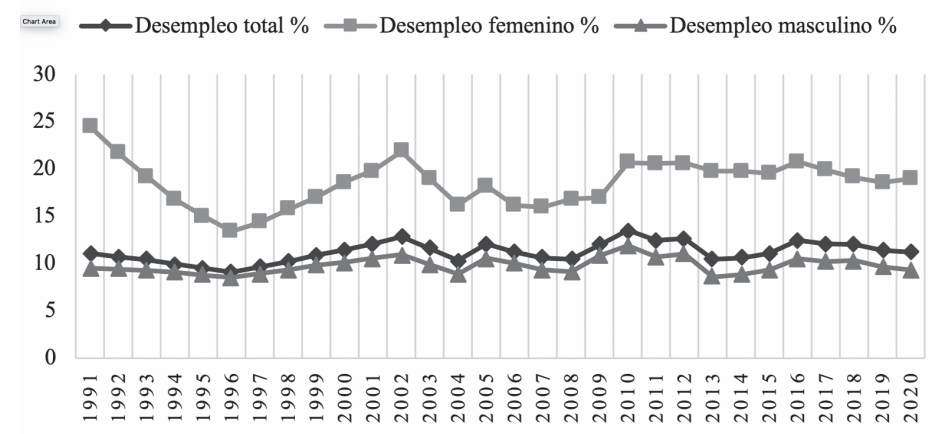

Elaboración propia con información de Banco Mundial, 2021.

Esta importante brecha de participación en el mercado laboral se ha producido en un contexto en el que las autoridades iraníes violan los derechos económicos y sociales de las mujeres, en conjunto con un modelo económico neoliberal implementado desde 
1989, que ha profundizado la desigualdad social y afecta en mayor medida a las mujeres (Sephri, 2017). Esta situación discriminatoria contra las iraníes no se debe únicamente a la incapacidad del mercado laboral para absorber el enorme ejército industrial de reserva, sino fundamentalmente a que el Estado ha impulsado políticas desfavorables para las mujeres en los ámbitos laboral, familiar, académico y profesional.

Ciertamente, desde el fin de la guerra con Iraq (1988), la República Islámica ha enfrentado una severa crisis de desempleo que se perfila como la mayor problemática del régimen. Si bien el desempleo afecta al grueso de la población iraní, son las mujeres las que recienten mucho más la pérdida de espacios laborales, ya que los empleadores deben adoptar una serie de normas y códigos de segregación en las fábricas, talleres y oficinas, así como solicitar el consentimiento por escrito de los tutores de las mujeres empleadas. Para evitar estos gastos, los empleadores prefieren contratar más hombres que a mujeres, con lo cual perpetúan la desigualdad económica y social en detrimento de las mujeres.

Hasta aquí se han detallado algunos elementos que a nivel interno influyen en la situación de las iraníes. Enseguida se desarrollarán aquellos que en el ámbito internacional han tenido un impacto en la vida de las mujeres iraníes.

\section{La Influencia de Factores Externos en la Situación de las Mu- jeres Iraníes: el Impacto de las Sanciones Económicas \\ Las sanciones estadounidenses contra Irán se remontan a la revo- lución de 1979 y la crisis de los rehenes. ${ }^{5}$ Sin embargo, el régimen de sanciones internacionales, que en la actualidad aísla a Irán, co- menzó en 2005 cuando se remitió el asunto del programa nuclear de Irán al Consejo de Seguridad de la Organización de las Nacio-}

\footnotetext{
5 Las primeras sanciones estadounidenses contra Irán siguieron a la captura de diplomáticos estadounidenses en Teherán, en 1979 (Samore, 2015, p. 3). El entonces presidente Jimmy Carter emitió una orden ejecutiva, en la que bloqueó las propiedades del gobierno iraní, incluido el Banco Central de Irán, además prohibió la venta y transporte de mercancías a Irán e impidió la concesión de créditos o prestamos a Irán (Samore, 2015, p. 36).
} 
nes Unidas (ONU). El bloqueo económico internacional contra Irán liderado por Estados Unidos se ha justificado bajo diversas narrativas, desde la proliferación de armas de destrucción masiva, el apoyo al terrorismo y la violación de los derechos humanos, a las que se recurren según la coyuntura global con el objetivo de roer la estructura del régimen iraní (Samore, 2015).

Las sanciones han provenido de tres actores: la Unión Europea, Estados Unidos y el Consejo de Seguridad de la ONU, aunque las más recientes fueron reimpuestas por el gobierno estadounidense en noviembre de 2018, luego de que en mayo del mismo año, Donald Trump decidiera cesar la participación de Estados Unidos del Plan de Acción Integral Conjunto (JCPOA, por sus siglas en inglés), ${ }^{6}$ puesto en vigor en julio de 2015.

De acuerdo con el Departamento del Tesoro de EU (2018), estas son las sanciones más duras que el país ha impuesto a Irán y se dirigen a sectores críticos de su economía, como la energía, el transporte marítimo, la construcción naval y los sectores financieros.

Si bien los bloqueos económicos y financieros tienen un fuerte impacto en la economía iraní, reflejado en la alta inflación, la pérdida de poder adquisitivo, el desempleo y la reducción en las exportaciones de petróleo; ${ }^{7}$ es la población quien más sufre los estragos en su día a día, sobre todo los jóvenes y las mujeres. Esto se observa en distintos ámbitos, como es el desempleo, cuya tasa durante el primer trimestre de 2019 se mantuvo notablemente alta, ya que en el grupo de edad de 15 a 29 años la tasa rondaba el $20.5 \%$ para hombres y $35.3 \%$ para las mujeres, lo que indica que las iraníes se encontraban entre las más afectadas (Kalhor, 2019).

6 El Plan de Acción Integral Conjunto (Joint Comprehensive Plan of Action) atiende a varios fines: establece límites detallados al desarrollo del programa nuclear iraní, cuyo cumplimiento se garantiza mediante un régimen de verificación, y pretende orientar el programa nuclear de Irán a fines comerciales y pacíficos mediante incentivos. Fue acordado por los cinco miembros del Consejo de Seguridad de la ONU, Irán y la Unión Europea (Antón, 2015).

Desde 2018, el rial iraní ha perdido el 68\% de su valor. En marzo de 2020, la inflación alcanzó alrededor del $41 \%$ y para 2021 ronda el 30\%. En el mismo período, el Producto Interno Bruto se redujo un 6.5\% y el desempleo se situó en el 10.8\% (Moaveni y Tahmasebi, 2021). 
Esto ha ocurrido pese a que, de acuerdo con el Índice Global de Brecha de Género realizado por el Foro Económico Mundial (2020), prácticamente existe una situación de paridad entre hombres y mujeres en la matriculación universitaria, ${ }^{8}$ de modo que las iraníes han utilizado la educación como un instrumento de agencia pese a que presentan una gran cantidad de obstáculos al intentar insertarse a la vida laboral. Según datos del Banco Mundial (2021a), se demuestra que en 2019 la tasa de participación en la fuerza laboral para hombres de entre 15 y 64 años era del 77\%, mientras que la de las mujeres en el mismo rango de edad apenas alcanzaba el $19 \%$.

Por otro lado, Azadeh Moaveni y Sussan Tahmasebi (2021) señalan que las vidas de las mujeres de clase media han sido afectadas por las sanciones estadounidenses en otros sentidos; por ejemplo, la falta de ingresos económicos y el aumento de los gastos, consecuencia de la recesión económica, han provocado que mujeres que vivían en entornos de violencia intrafamiliar se vean orilladas a regresar al lugar donde sufrieron las condiciones de abuso.

Asimismo, la labor de las activistas iraníes ha sido afectada por las sanciones debido a que el gobierno iraní ha adoptado una estrategia de seguridad más severa hacia la sociedad civil (Tahmasebi, 2018). ' Las facciones conservadoras, hasta cierto punto, se han beneficiado del bloqueo internacional debido a que les permite fortalecer su retórica antiimperialista y afianzarse en los órganos de mayor autoridad de la República. En consecuencia, los grupos conservadores impulsan nuevas campañas sobre la vestimenta de las mujeres y su conducta en lugares públicos.

\footnotetext{
8 Los valores del Índice Global de Brecha de Género van de 1 (igualdad) a 0 (desigualdad). El puntaje de la matriculación universitaria es de 0.86 (Foro Económico Mundial, 2020).

9 Las organizaciones de derechos humanos Human Rights Watch y Amnistía Internacional han reportado detenciones como la de la abogada y activista Nasrin Sotoudeh, quien fue encarcelada en 2018 acusada de espiar, difundir propaganda e insultar al Líder Supremo de Irán.
} 
174 UNA APROXimaCión a LA SiTUACión DE LAS MUJERES EN EL IRÁN CONTEMPORÁNEO: PERSPECTIVAS locales y Globales / Alejandra Gutiérrez Luna, Iraís Fuentes Arzate

Dado que las sanciones estadounidenses afectan la economía del país, las autoridades mantienen un estricto control sobre las reuniones pacíficas, en particular las protestas relacionadas con la falta de empleos (Human Rights Watch, 2020). Incluso, algunas defensoras de los derechos de las mujeres han sido acusadas de tratar de adoptar comportamientos occidentales que socavan los valores islámicos del país. Las sanciones y el aislamiento de Irán han impactado constantemente a las activistas y su capacidad para conectarse con sus contrapartes extranjeras por el aislamiento que sufren al interior del país (Tahmasebi, 2018).

El Centro de Derechos Humanos en Irán lanzó un comunicado en 2019, en el cual destaca que quienes dentro del gobierno estadounidense defienden la aplicación de las sanciones en nombre de los derechos humanos esperan que la presión sobre el pueblo iraní provoque un levantamiento popular o una revolución, pero no comprenden que el hecho de debilitar al gobierno no daría como resultado automático el fortalecimiento de la sociedad civil. De hecho, la sociedad civil es una de las principales víctimas de estas políticas.

En este sentido, las estrategias de política exterior impulsadas por Estados Unidos utilizan a las mujeres como instrumento para desprestigiar al régimen iraní, pues aunque es de conocimiento de los gobiernos estadounidenses que las sanciones pueden afectar a la población iraní, y en especial a las mujeres, continúan con su implementación. Así, por un lado existe un discurso de apoyo a los derechos humanos de las mujeres iraníes, el cual puede ilustrarse con dos ejemplos: primero, en mayo de 2018, el entonces Secretario de Estado, Mike Pompeo, lanzó la "Nueva Estrategia de Irán" ante la Fundación Heritage. ${ }^{10}$ En sus comentarios, insistió en que la larga lucha de las mujeres iraníes por la inclusión y la igualdad es muy importante para Washington (Moaveni y Vaez, 2020), mientras que dejaba claro que Esta-

${ }^{10}$ La Fundación Heritage una organización cuya misión es formular y promover políticas públicas conservadoras basadas en los principios de libre empresa, gobierno limitado, libertad individual, valores estadounidenses tradicionales y una defensa nacional sólida. 
dos Unidos aumentaría la presión sobre Irán y "el mundo podía aceptarlo o no" (Rogin, 2018).

Adicionalmente, en enero de 2020 el Departamento de Estado publicó en Twitter un video sobre la historia de los derechos de las mujeres iraníes, en el que se muestran mujeres con la cabeza descubierta, aludiendo a una época en la que las iraníes "disfrutaban de libertad e igualdad de oportunidades", antes de pasar a imágenes oscuras de 1979 cuando, de acuerdo con el video, la revolución islámica "provocó un retroceso en la situación de las iraníes" (Moaveni y Vaez, 2020). Por otro lado, y en contraste con este discurso, las sanciones estadounidenses desmantelan la economía de un país y el sustento de su población, al tiempo que afectan a uno de los motores del cambio social en Irán: las mujeres (Moaveni y Tahmasebi, 2021).

Aunado a lo anterior, las perspectivas económicas de Irán dependen de la evolución de la pandemia de covid-19 y del ritmo de la recuperación económica mundial. Se prevé que la recuperación de su PIB sea gradual, debido a la lenta implementación de la vacunación, y es probable que la inflación se mantenga, en promedio, por encima del $20 \%$ a mediano plazo, por lo que continuará la presión económica sobre los hogares pobres (Banco Mundial, 2021).

\section{Las agendas internacionales en torno de la cuestión de las mujeres}

El régimen internacional de protección de derechos humanos se compone por numerosos instrumentos internacionales, entre los que se encuentran tratados, convenciones y protocolos. Algunos de estos instrumentos se relacionan de manera explícita con categorías amplias, pero particulares de derechos, como los derechos civiles y políticos o los económicos, sociales y culturales. Otros conjuntos de normas y órganos se pueden agrupar en torno a los sujetos a los cuales buscan proteger, como las mujeres, los niños y niñas, los trabajadores migratorios o las personas con discapacidad (Anaya, 2017). 
176 UNA APROXimaCión a LA SITUACión DE LAS MUJERES EN EL IRÁN CONTEMPORÁNEO: PERSPECTIVAS locales y Globales / Alejandra Gutiérrez Luna, Iraís Fuentes Arzate

En este sentido, se puede hacer referencia a un régimen internacional de los derechos de las mujeres. Este se compone de principios, normas, reglas y procedimientos de toma de decisiones en la materia. El régimen incluye actores gubernamentales y no gubernamentales, los cuales se pueden agrupar en organismos intergubernamentales (OIG) y organizaciones no gubernamentales (ONG). Entre los primeros destaca el trabajo de la ONU, que es el organismo en el que se enfoca este escrito.

Los principios del régimen internacional de los derechos de las mujeres se encuentran articulados en varios instrumentos, los cuales incluyen mecanismos de supervisión y monitoreo para formular y hacer cumplir las reglas, incluida una variedad de comisiones, tribunales y grupos de expertos que monitorean el cumplimiento y pueden aplicar sanciones (Kardam, 2002, p. 416). Los órganos de la ONU, cuyo desempeño se basa en los asuntos de los derechos de las mujeres, son la Comisión de la Condición Jurídica y Social de la Mujer ${ }^{11}$ y ONU Mujeres.

El primero se trata del principal órgano internacional intergubernamental dedicado exclusivamente a la promoción de la igualdad de género y el empoderamiento de las mujeres. La comisión depende del Consejo Económico y Social, y se reúne cada año con el objetivo de que los representantes de los Estados Miembros de las Naciones Unidas, las organizaciones de la sociedad civil y las entidades de las Naciones Unidas debatan sobre los avances y la aplicación de la Declaración y Plataforma de Acción de Beijing de 1995 y la 23a Sesión Especial de la Asamblea General, celebrada en el año 2000 (Beijing +5), así como sobre las cuestiones emergentes que afectan la igualdad de género (ONU Mujeres, 2021). Por su parte, ONU Mujeres apoya a los Estados Miembros de las Naciones Unidas en el establecimiento de normas internacionales para lograr la igualdad de género y el respeto de sus derechos.

${ }^{11}$ En abril de 2021, el Consejo Económico y Social de la ONU eligió a Irán para presidir por los siguientes cuatro años la Comisión de la Condición Jurídica y Social de la Mujer, lo cual desató una gran controversia, dadas las condiciones en las que viven las iraníes al interior del país, producto en gran medida de las duras y discriminatorias leyes en materia civil y penal. 
El marco institucional bajo el que operan ambos órganos es la Convención sobre la Eliminación de Todas las Formas de Discriminación contra la Mujer (CEDAW), adoptada en forma unánime por la Asamblea General de la ONU el 18 de diciembre de 1979. Se trata de la carta internacional de los derechos de las mujeres y provee un marco obligatorio de cumplimiento para los países que la han ratificado en aras de lograr la igualdad de género (ONU Mujeres, 2011).

Es precisamente allí donde radica una de las objeciones por parte de Estados que se niegan a ratificar la convención o lo hacen con reservas, ya que no existe un acuerdo claro entre los Estados sobre lo que constituye discriminación e igualdad. Algunos sostienen que los roles de género asignados por motivos culturales y religiosos no constituyen discriminación. Otros argumentan que las reservas respecto a la convención reflejan una discriminación sistemática contra las mujeres basada en un modelo de opresión patriarcal y, por lo tanto, son contrarias al objeto y propósito de la CEDAW (Kardam, 2002).

En el caso de Irán, en 1996, durante la presidencia de Akbar Hashemí Rafsanyaní (1989-1997), el Ministerio de Relaciones Exteriores se encargó de revisar la CEDAW y consideró que algunos de sus artículos contradecían distintas leyes internas contenidas en la Constitución y en los códigos civil y penal, por lo que formularon algunas reservas. Un año después, la convención fue revisada por el Consejo Supremo de la Revolución Cultural, el cual concluyó que contradecía la sharia en 40 casos y estaba en contra de leyes nacionales en 70 casos. Fue así como este consejo rechazó contundentemente la ratificación de la CEDAW (Women's Committee of the National Council of Resistance of Iran, 2016).

Posteriormente, en 1999, bajo la presidencia de Mohammad Jatamí, el gobierno iraní presentó un informe ante la ONU, indicando que se había asignado a un grupo de trabajo la tarea de estudiar la posibilidad de unirse a la convención, y tras realizar una consulta con varios ministerios, un subcomité gubernamental finalmente decidió, en diciembre de 2001, unirse a la CEDAW. 


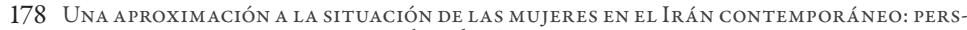
PECTIVAS locales y Globales / Alejandra Gutiérrez Luna, Iraís Fuentes Arzate

Sin embargo, el Consejo de Guardianes rechazó la decisión bajo el argumento de que la convención era incompatible con algunos principios del islam, específicamente respecto a temas como las herencias, el divorcio, la poligamia y la vestimenta (Women $>$ s Committee of the National Council of Resistance of Iran, 2016).

En este sentido, el Líder Supremo Alí Jameneí consideró que la aceptación de las prescripciones occidentales de la CEDAW estaría en contra de la dignidad islámica y sería contraproducente, pues el régimen tendría que rendirse a las instrucciones de las "potencias arrogantes" y respaldarlas (Women's Committee of the National Council of Resistance of Iran, 2016).

En 2013, durante su campaña presidencial, Hasán Ruhaní prometió ratificar la CEDAW. De hecho, en su primera conferencia de prensa como presidente, destacó que el objetivo en el tema de las mujeres debía ser eliminar todo "comportamiento primitivo" que inhiba una mayor participación femenina en la vida pública. No obstante, la mayoría conservadora en el Parlamento actual $^{12}$ se ha convertido en un obstáculo para el avance en la ratificación de la convención (Alikarami, 2014). En resumen, son las características del sistema político iraní las que han impedido un avance en la ratificación de la CEDAW.

Algunos temas en los que se encuentran contradicciones entre las leyes iraníes y la CEDAW son, por ejemplo, la edad mínima para contraer matrimonio, la cual en 2002 pasó de 9 a 13 años para las niñas de acuerdo con el Código Civil, aunque el padre o el abuelo paterno pueden casar a las niñas antes de la edad legal. Esto contrasta con el artículo 16 de la CEDAW que prohíbe el matrimonio infantil (Bakhshizadeh, 2018).

Por otro lado, el artículo 1105 del Código Civil iraní reconoce "la posición de la cabeza de familia como derecho exclusivo del marido", mientras que el artículo 5 de la CEDAW pide la modificación de los patrones sociales y culturales de conducta de hom-

\footnotetext{
${ }^{12}$ Durante las elecciones legislativas de febrero de 2020, los partidos conservadores y ultraconservadores consiguieron 221 de los 290 escaños del Parlamento. La alianza de reformistas y centristas apenas lograron 19 (Espinosa, 2020).
} 
bres y mujeres basados en una supuesta inferioridad o superioridad de uno u otro sexo, o en roles estereotipados para hombres y mujeres (Bakhshizadeh, 2018).

De igual manera, las mujeres iraníes no tienen el mismo derecho a la disolución del matrimonio. El artículo 1133 del Código Civil otorga al hombre el derecho a divorciarse de su esposa siempre que lo desee, pero la mujer no tiene el mismo derecho. Según el artículo 1130 de la constitución iraní, las mujeres solo lo pueden hacer en determinadas circunstancias (Bakhshizadeh, 2018). Por su parte, el artículo 16 de la CEDAW insiste en que deben adoptarse medidas adecuadas para eliminar la discriminación contra las mujeres en todos los asuntos relacionados con el matrimonio.

Así pues, la desigualdad de género se encuentra institucionalizada, y más allá de que exista el argumento acerca de que los OIG como ONU Mujeres intentan universalizar su perspectiva sobre los derechos de las mujeres, es evidente que las leyes iraníes mencionadas afianzan el sistema de dominación patriarcal y la desigualdad entre hombres y mujeres.

\section{Los feminismos trasnacionales}

Otro de los factores internacionales que ha tenido un impacto en la condición de las mujeres iraníes se relaciona con los mecanismos de lucha de distintos movimientos feministas alrededor del mundo, en específico las redes de sororidad trasnacional, las cuales consolidan a las mujeres como actores en la escena internacional y hacen visibles sus experiencias.

El movimiento de mujeres iraníes se ha convertido en uno de los más importantes en las sociedades islámicas y en el mundo. Las iraníes han luchado por reformas legislativas, políticas y democráticas, y han logrado importantes cambios, en especial en el ámbito del derecho familiar. Asimismo, han desempeñado un papel crucial en el fomento de una sociedad civil donde las mujeres lideran y trabajan en organizaciones independientes y asociaciones civiles (Povey, 2021, p. 169). Por ejemplo, con la campaña 
180 UNA APROXIMACIÓN A LA SITUACIÓN DE LAS MUJERES EN EL IRÁN CONTEMPORÁNEO: PERSPECTIVAS LOCAlES y GlobAles / Alejandra Gutiérrez Luna, Iraís Fuentes Arzate

"Un Millón de Firmas para la Eliminación de Leyes Discriminatorias", activistas iraníes dieron seguimiento a la protesta pacífica que tuvo lugar en junio de 2006 en la plaza Haft-e Tir de Teherán. Las firmantes de la campaña, en representación de grupos y activistas internacionales de mujeres, centros universitarios y académicos, expresaron su apoyo a las mujeres iraníes en su esfuerzo por reformar las leyes y lograr un estatus igualitario dentro de la legislación iraní (Change for Equality, 2006).

Una de las organizaciones de la diáspora iraní más activas en cuestiones de mujeres es la Fundación de Estudios de la Mujer Iraní (IWSF, por sus siglas en inglés), cuyo objetivo desde 1990 ha sido ha sido el intercambio de ideas sobre cuestiones relacionadas con las mujeres (Ghorashi y Tavakoli, 2006). Difunde información sobre los logros de las iraníes y ha establecido una red de comunicación entre las comunidades de mujeres iraníes alrededor del mundo. Desde la perspectiva de esta organización, la mayor parte de la historia de Irán ha consistido en narrativas dominadas por hombres y relatos de las hazañas y logros de los hombres, por lo que la IWSF intenta ofrecer una perspectiva diferente sobre la historia de Irán, protegiendo, preservando y difundiendo las obras de las mujeres iraníes, y desde su creación ha realizado conferencias anuales en varias ciudades del mundo (Iranian Women's Studies Foundation, 2021).

Igualmente, sobresalen activistas pro-derechos humanos, cuya lucha ha sido notable y reconocida a nivel internacional, como Narges Mohammadi, sentenciada a 11 años de prisión en octubre de 2011 por cargos de "reunión y colusión contra la seguridad nacional", "pertenencia al Centro de Defensores de Derechos $\mathrm{Hu}$ manos" y "propaganda contra el Estado”. Fue liberada en 2012. Sin embargo, en mayo de 2015 fue denunciada por "actividades contra la seguridad nacional y propaganda antigubernamental”. Finalmente fue puesta en libertad en octubre de 2020 (Center for Human Rights in Iran, 2015).

El movimiento de mujeres ha luchado por liberarse del gobierno autocrático, pero también contra la dominación y el imperialismo occidental (Povey, 2021); por ejemplo, contra las represen- 
taciones del islam como una religión atrasada y antidemocrática, utilizadas por ciertos gobiernos, como el estadounidense, para justificar un régimen de sanciones internacionales que ha perjudicado a las iraníes y, al mismo tiempo, ha fortalecido a las facciones más conservadoras de Irán (Povey, 2021).

Como señala Nayereh Tohidi (2016), la globalización ha intensificado una dialéctica glocal, es decir, la interacción de factores locales y globales. El dinamismo glocal de la sociedad iraní se ha fortalecido, particularmente en las últimas cuatro décadas, debido al impacto de las personas exiliadas, en su mayoría asentados en Europa Occidental y América del Norte. Este éxodo masivo de iraníes ha resultado en la formación de comunidades que incluyen a profesionales con altos niveles educativos, así como activistas feministas que han constituido uno de los componentes más importantes de las conexiones trasnacionales y el proceso glocal de cambios socioculturales en Irán.

Justamente, Nayereh Tohidi, profesora e investigadora iraní de la Universidad Estatal de California, Northridge (2021) ha combinado su activismo trasnacional en asuntos de derechos humanos con el trabajo académico. Ha escrito artículos sobre género, feminismo y globalización en Irán, además de haber sido consultora de la ONU en proyectos sobre género y desarrollo. Su activismo transnacional ha incluido la representación de organizaciones de mujeres en la tercera y cuarta Conferencias Mundiales de la ONU sobre la Mujer en Nairobi (1985) y Beijing (1995).

Finalmente, pese a que en Irán existen serias restricciones en el acceso a internet, algunas mujeres han aprovechado esta herramienta de comunicación, ya que es imposible para el Estado controlar el ciberespacio por completo, lo que lo convierte en un medio poderoso para las conexiones transnacionales en apoyo a las protestas locales (Ghorashi \& Tavakoli, 2006).

El internet ha habilitado localmente a activistas para entrar en un espacio virtual trasnacional. Ha habido muchos movimientos de protesta en internet contra la violencia doméstica o pública contra las mujeres en Irán; se difunden noticias sobre mujeres torturadas o golpeadas por sus maridos o tratadas con crueldad 
en los tribunales o cárceles; como ocurrió cuando se propagó internacionalmente el caso de la periodista Zahra Kazemi, quien en 2004 fue torturada y asesinada en una prisión iraní (Ghorashi y Tavakoli, 2006). De igual forma, durante 2020, algunas mujeres iraníes compartieron en Twitter sus historias de acoso, agresión y abuso sexual. Las publicaciones utilizaban hashtags en farsi que se traducen como "no significa no" y "no a la violación" (Jaafari, 2020).

\section{Reflexiones finales}

A lo largo de este artículo se desarrollaron algunas perspectivas locales y globales que han tenido un impacto en la situación de las mujeres en Irán durante la década más reciente. Es posible afirmar que, más allá de consideraciones estereotipadas sobre las mujeres iraníes que consideran al islam per se y a la imposición del velo como las fuentes de opresión más sobresalientes ante las cuales las mujeres son solo víctimas; existen factores como la Ley de la Familia, las características del sistema político iraní, el desempleo, el predominio del sistema patriarcal, e incluso factores externos como las sanciones económicas impuestas por el gobierno estadounidense, que han sido un obstáculo para la mejora de las condiciones de vida de las iraníes. Adicionalmente, los altos niveles educativos, las agendas de los organismos internacionales en torno a los derechos de las mujeres y la influencia de los movimientos feministas trasnacionales, se han convertido en elementos que algunas mujeres han empleado para tener una mayor participación y visibilidad en la sociedad y en la política.

La situación de las mujeres en Irán no se limita a las estructuras patriarcales que impiden cambios favorables a la igualdad de género; también se caracteriza por la actividad política de las iraníes para conseguir mayores libertades y derechos civiles. Frente al activismo de las mujeres, el régimen islámico ha adoptado nuevos métodos de represión y censura contra las mujeres, extendido al uso de tecnología para dispersar manifestaciones, tortura y espionaje, así como la ampliación del cuerpo de los Basiy para man- 
tener el control de la organización de mujeres en universidades, plazas públicas, mezquitas y estadios de fútbol como métodos de contención de la protesta social; en consecuencia, en la última década las mujeres han sido reprimidas como nunca.

A pesar de esto, las organizaciones de mujeres iraníes no han descansado, sino que han llevado su política a los "espacios clandestinos". Las mujeres están utilizando internet y las redes sociales de manera más activa que cualquier otro grupo social para abordar problemas relacionados con los roles de género y las prácticas patriarcales convencionales (Koo, 2016). El uso extensivo de redes sociales como medio de organización social y de manutención de los lazos de sororidad transnacional representan un cambio significativo en las organizaciones de mujeres, ya que permite un alcance y difusión mayor de las actividades realizadas por sus colectivas.

\section{Referencias}

Alikarami, L. (2014, 18 de diciembre) CEDAW and the quest of Iranian women and gender equality. Open Democracy. https:// www.opendemocracy.net/en/5050/cedaw-and-quest-of-iranian-women-for-gender-equality/

Anaya, A. (2017). Los regímenes internacionales de derechos humanos: la brecha entre compromiso y cumplimiento. Revista del Instituto de Ciencias Juridicas de Puebla, 11(40), 159-181. http://www. scielo.org.mx/pdf/rius/v11n40/1870-2147-rius-11-40-00159.pdf Aryan, K. (2016). The Boom in Women's Education. En Women, Power and Politics in 21st century Iran. Routledge.

Antón, S. (2015, 21 de octubre) Vigilancia y limites al programa nuclear de Irán: el Plan de Acción Integral Conjunto (Joint Comprehensive Plan of Action, JCPOA). Real Instituto Elcano. https://tinyurl.com/3jpndwra

Bahramitash, R. (2007). Iranian women during the reform era (1994-2004). A focus on employment. Journal of Middle East Women's Studies, 3(2). 86-109.

Bahramitash, R. \& Esfahani, H. (2011). Modernization, Revolution, and Islamism. Political Economy of Women's Em- 
184 UNA APROXIMACIÓN A LA SITUACIÓN DE LAS MUJERES EN EL IRÁN CONTEMPORÁNEO: PERSPECTIVAS locales y Globales / Alejandra Gutiérrez Luna, Iraís Fuentes Arzate

ployment. En Veiled Employment. Islamism and the political economy of women's employment in Iran. Syracuse University Press.

Bakhshizadeh, M. (2018) Changing Gender Norms in Islam Between Reason and Revelation. Budrich UniPress.

Banco Mundial. (2021). Islamic Republic of Iran. Overview. https://www.worldbank.org/en/country/iran/overview\#1

Banco Mundial. (2021a). Tasa de participación en la fuerza laboral. https://datos.bancomundial.org/indicator/SL.TLF. ACTI.FE.ZS?locations $=$ IR

Banco Mundial. (2021b). Unemployed male. https://data.worldbank.org/indicator/SL.UEM.TOTL.MA.ZS?locations=IR

Center for Human Rights in Iran. (2015, 19 de octubre). Narges Mohammadi Should Be Released Immediately and Given MedicalTreatment.https://www.iranhumanrights.org/2015/10/ narges-mohammadi-8/

Change for Equality. (2006, 27 de agosto). Petition: International Support for Women's Campaign. https://www.we-change. org/english/spip.php?article19

Departamento del Tesoro de Estados Unidos (2018). Re-imposition of the sanctions on Iran that had been lifted or waived under the JCPOA. https://tinyurl.com/fw97a9s

Espinosa, A. (2020, 26 de febrero). Los conservadores se consolidan en Irán en unas elecciones con una elevada abstención. El Pais. https://elpais.com/internacional/2020/02/26/actualidad/1582737801_978726.html

Foro Económico Mundial. (2020). Informe Mundial de Brecha de Género. https://www.weforum.org/reports/gender-gap-2020-report100-years-pay-equality

Ghorashi, H. \& Tavakoli, N. (2006). Paradoxes of transnational space and local activism: Iranians organizing across borders. Focaal-European Journal of Anthropology, 47, 90-102. https://tinyurl.com/39af45kd

Golkar, S. (2015). Captive Society. The Basij Militia and Social Control in Iran. Columbia University Press. 
Human Rights Watch. (2020). Iran. Events of 2019. https:// www.hrw.org/world-report/2020/country-chapters/iran\#

Iranian Women's Studies Foundation. (2021). Statement of Purpose. https://www.iwsf.org/about-us

Jaafari S. (2020, 3 de septiembre). Iranians shares stories of sexual harassment, abuse on social media. The World. https://www. pri.org/stories/2020-09-03/iranians-share-stories-sexual-harassment-abuse-social-media

Kalhor, N. (2019, 15 de agosto). Improved employment leaves Iran's women, youth behind. Al-Monitor. https://www. al-monitor.com/originals/2019/08/iran-improved-employment-women-youth-left-behind.html

Kar, M. \& Farshi, F. (2008). Focusing on Women in the Internal Politics of Iran. The Brown Journal of World Affairs, 15(1). https://www.jstor.org/stable/24590950?seq=1\#metadata info_tab_contents

Kardam, N. (2002). The Emergence of a Global Gender Equity Regime. International Journal, 57(3), 411- 438.

Kian, A. (2005). From motherhood to equal rights advocates: The weakening of patriarchal order. Iranian Studies, 38(1). https://www.jstor.org/stable/4311705?seq=1

Kian, A. (2012). Gendered citizenship and the women's movement in Iran. En Iran: a revolutionary republic in transition. European Union Institute for Security Studies.

Kian, A. (2013). Social and cultural change and the women's rights movement in Iran. En Cultural revolution in Iran. Contemporary Popular Culture in the Islamic Republic. Tauris.

Koo, G. Y. (2016). To be Myself and have My Stealthy Freedom: The Iranian Women's Engagement with Social Media. Revista de Estudios Internacionales Mediterráneos, 21. http://dx. doi.org/10.15366/reim2016.21.011

Mir-Hosseini, Z. (1993). Women, Marriage and the Law in Post-Revolutionary Iran. En Women in the Middle East. Perceptions, Realities and Struggles for Liberation. Palgrave Macmillan. 
186 UNA APROXiMACión A LA SITUACión DE LAS MUJERES EN EL IRÁN CONTEMPORÁNEO: PERSPECTIVAS locales y Globales / Alejandra Gutiérrez Luna, Iraís Fuentes Arzate

Moaveni, A. \& Tahmasebi, S. (2021, 27 de marzo). The Middle-Class Women of Iran Are Disappearing. The New York Times. https://www.nytimes.com/2021/03/27/opinion/sunday/ iran-sanctions-women.html

Moaveni, A. \& Vaez, A. (2020, 6 de marzo) U.S. 'Maximum Pressure' on Iran Hurts the Women It Claims to Help. Crisis Group. https://tinyurl.com/292nf7zz

Navas, M. (2018). Equidad de género: una aproximación histórica a las políticas públicas para la equidad de género en $\mathrm{El}$ Salvador (2017). En Antología del pensamiento crítico salvadoreño contemporáneo (pp. 347-366). CLACSO. doi:10.2307/j. ctvfjd0vt.15

Nayereh, T. (2016). Women's rights and feminist movements in Iran. Sur 24, 13(24), 75-89. https://papers.ssrn.com/sol3/papers.cfm?abstract_id=3028205

Nejadbahram, Z. (2016). Women and Employment. En Women, Power and Politics in 21st century Iran. Routledge.

ONU Mujeres. (2011). Convención sobre la Eliminación de Todas las Formas de Discriminación contra la Mujer. https://tinyurl. com/2h6yc395

ONU Mujeres. (2021). Comisión de la Condición Jurídica y Social de la Mujer. https://www.unwomen.org/es/csw

Osanloo, A. (2009). The Politics of Women's Rights in Iran. Princeton University Press.

Povey, T. (2016). The Iranians Women's Movement in its Regional and International Context. En Povey, T. \& Rostami-Povey, E. (Ed.) Women, Power \& Politics in 21st Century in Iran. Routledge. Rogin, J. (2018, 21 de mayo) Opinion: Pompeo's Iran strategy speech lacked a real strategy. The Washington Post. https://www. washingtonpost.com/news/josh-rogin/wp/2018/05/21/pompeos-iran-strategy-speech-lacked-a-real-strategy/

Sadeghi, F. (2009). Foot Soldiers of the Islamic Republic's 'Culture of Modesty'. Middle East Report, The Islamic Revolution, 250. http://www.jstor.com/stable/27735286.

Sephri, T. (2017). "It's a Men's Club” Discrimination Against Women in Iran's Job Market. Human Rights Watch. https:// 
www.hrw.org/report/2017/05/25/its-mens-club/discrimination-against-women-irans-job-market

Sreberny, A. (2013). Thirty-plus years of the Iranian Revolution: Culture in contestation. En Cultural revolution in Iran.Contemporary Popular Culture in the Islamic Republic. Tauris.

Tahmasebi, S. (2018, 10 de agosto). How US Sanctions Impede the Women's Movement in Iran. Atlantic Council. https:// www.atlanticcouncil.org/blogs/iransource/how-us-sanctions-impede-the-women-s-movement-in-iran/

Tajali, M. (2015). Islamic Women's Groups and the quest for Political Representation in Turkey and Iran. Middle East Journal, 69(4). doi.org/10.3751/69.4.14

Universidad Estatal de California, Northridge. (2021). Nayereh Tohidi. https://www.csun.edu/humanities/gender-womens-studies/nayereh-tohidi

Vakil, Sanam. (2011). Women and politics in the Islamic Republic of Iran. Action and reaction. The Continuum International Publishing Group.

Yassari, N. (2002). Iranian Family Law in Theory and Practice. Yearbook of Islamic and Middle Eastern Law Online, 9(1). https://doi.org/10.1163/22112987-91000006

Women's Committee of the National Council of Resistance of Iran. (2016). Why the Iranian Regime Does Not Join the CEDAW? https://women.ncr-iran.org/2016/03/05/why-the-iranian-regimedoes-not-join-the-cedaw/

World Economic Forum. (2020). Global Gender Gap Report 2020. World Economic Forum. World Economic Forum. https://www.weforum.org/reports/gender-gap-2020-report100-years-pay-equality

Zaccara, L. (2006). Los enigmas de Irán. Sociedad y política en la República Islámica. Capital Intelectual. 